\title{
Psychosocial Support Provided by Health Volunteer for Older Adult with Depression and Suicide Attempt: A Case Study
}

\author{
Saifon AEKWARANGKOON*, Naiyana NOONIL and Rewwadee PETSIRASAN
}

School of Nursing, Walailak University, Nakhon Si Thammarat 80161, Thailand

('Corresponding author's email: asaifon@wu.ac.th)

Received: 31 May 2017, Revised: 15 April 2018, Accepted: 19 May 2018

\begin{abstract}
Depression causes major disabilities and can lead to suicide among older adults in Thai rural communities. A case study was used to present the psychosocial support provided by a health volunteer to an older adult suffering from depression and attempted suicide. Content analysis was applied to analyze the data. The results revealed that the psychosocial support given by the health volunteer consisted of 4 processes; 1) support and motivation to keep alive, 2) encouragement for a new life, 3) empowerment for life change, and 4) evaluation of the change process. These processes not only help mitigate patients' symptoms of depression and possible suicide risks but also prove to promote psychological well-being. The patient outcome benefits indicated sustainability over the course of 18 months.
\end{abstract}

Keywords: Depression, suicide attempt, psychosocial support, health volunteer, older adult

\section{Introduction}

Depression is a major cause of suicide experienced by older adults and has increased annually across the world [1,2]. Older adults have been identified as the group with the highest possible risk of depression and suicide from life crises and health status [3,4]. Suicide as a result of depression ranks as the leading cause of death, especially among people aged 70 years and over [4,5]. The Thai government has invested financially to solve these problems [6]. However, the prevalence of undetected and untreated depression and suicide still intensifies among older adults [1,5].

In Thailand, depression and suicide is a culturally sensitive topic difficult to discuss among older adults [5-7]. Many older adults are concerned with not only the possibility of social disapproval on their mental health status, but also potential stigmatizing effects on them and their family [8-9]. In addition, depression and suicide is perceived as a sensitive topic among many health care providers, causing difficulties for a heartfelt discussion with older adults and their families [10]. Furthermore, insufficient manpower in healthcare personnel is also an impactful deterrent in the Thai rural community $[6,10]$. Healthcare providers are compelled to halfheartedly carry out or completely ignore screening and interventions. As a result, this setback can restrain the flow of accurate information concerning older adults' mental health status, which can trigger mental health and psychiatric problems while, based on fundamental human rights, older adults are entitled to obtain information about mental health and life [1011].

Substantial literature conducted over the last half-century has emphasized the importance of early suicide screening among older adults with depression as well as interventions to prevent loss among older adults in the rural community [6,7]. Routine screening for depression and suicide, early effective interventions, and continuing care for depression and suicide among older adults in the rural community is strongly recommended [7]. Older adults receiving early depression screening and interventions, and continuing care run a lower risk of suicide than those receiving only suggestions or no treatment $[11,2]$. Much evidence indicates that effective early screening and interventions such as psychosocial support and 
http://wjst.wu.ac.th

counseling in a community based on a non-stigmatizing and positive approach has been associated with life-energy components including self-esteem, making choices, responsibility, and self-congruence also bolstered health care provider-older adult relationship that leads to decreased age involvement in mental health and psychiatric problems including suicide [1,7]. Early screening and interventions is also intended to provide older adults with attitude, information, and skills needed to maintain good health [7].

Research is required to illuminate stakeholders including healthcare personnel, health volunteers, and older adults' family on what older adults need and how to best reach out to them to encourage responsible mental health and empower them to prevent and promote their mental health [7]. Although there are research findings indicating strong correlation between depression, suicide, and older adults $[2,13]$, in Thailand the guideline for standard care does not yet include early screening and interventions all older adults for depression and suicide $[1,10]$. Under these circumstances, health volunteers play a significant role in preventing depression and suicide in the rural community despite limitations in health care manpower that is linked with better mental health, physical health, and health behaviors [10,14]. Many evidenced-based studies identified that professionally trained volunteers could benefit communities in helping older persons avoid the need for daily nursing care [15]. This paper particularly seeks to address this issue by proposing a complementary intervention of psychosocial support for depression and suicide provided by the health volunteer in a case study analysis of a Thai male patient with depressive symptoms and suicide risks in a rural community exhibiting a high rate of suicide from depression among older adults.

\section{Materials and methods}

\section{Participants}

Two participants were the patient, referred to as "V" and the health volunteer, referred to as "trained HV" living in Praduhom community, Thasala district, Nakhon Si Thammarat province, Thailand. This trained HV had succeeded in assisting with complicated cases of depression and suicide risks.

$\mathrm{V}$ is a 67-year-old male diagnosed with depression. His most troubling initial symptoms were sadness, loneliness, feeling of burden, low self-esteem, low energy, insomnia, reduced socialization, crying, helplessness, worthlessness, and attempted suicide. Causes of trauma include a bankrupt family business and the death of his wife 5 years ago. He lived with his son and was constantly consumed by the thought that he was worthless and also a burden to his son. He developed severe symptoms of depression including loss of interest in pleasurable activities, staying in bed all day, excessive crying, and suicide attempts. Prior to his family' business bankruptcy, he had been able to keep regular medical appointments for an annual checkup but he lived an unhealthy lifestyle (always experienced extreme stress, no exercise) and did not relax. Withdrawal, isolation, introjection, and repression were his defense mechanisms. Isolation was his primary form of coping for relaxation.

V's symptoms of depression and suicidal thoughts were detected by a trained HV in the first home visit. While $\mathrm{V}$ was contemplating suicide by hanging in his bedroom, the trained $\mathrm{HV}$ assigned to take care of the elderly with depression in the community and paid him a visit. The sudden approach of the trained $\mathrm{HV}$ includes active listening, concern questioning, and empathy that earned trust from $\mathrm{V}$. Then, he confided to the trained $\mathrm{HV}$ his suicide plan. Upon acknowledgement, the trained $\mathrm{HV}$ employed the process of psychosocial support and referred $\mathrm{V}$ to a nurse practitioner. After treatment, the trained HV still continued to provide psychosocial supports. During 6 months, V had become depressed and been helped by the trained HV. V was motivated to change his thoughts, perceptions, emotions, behaviors and achieve better management of his illnesses. He cooperated with trained HV focused on his depression and suicide.

\section{Background of psychosocial supporting older adult with depression by trained HV in community}

Health volunteers were trained by the researcher, the first author who is a depression specialist in the health care center in charge of screening and providing psychosocial support to older adults with depression and suicidal thoughts. The 2 week training course emphasized creating a positive attitude, knowledge, and skills on how to conduct an effective depression screening using PHQ-9, assessment of 
http://wjst.wu.ac.th

depression and how to detect early suicidal signs using PHQ-8, assessment of suicide among older adults with depression without stigmatizing them by giving psychosocial support, training process focused on how to actively listen, using concern questions to explore self-understanding, empowering, and providing resources on health. Trained HVs needed to pass the test before starting the psychosocial support process in the real situation and they may refer severely depressed older adults to the nurse practitioner.

\section{Interview}

For this descriptive case study the researcher team conducted a series of in-depth interviews for $\mathrm{V}$ and the trained HV who took care of V. The interview was designed to assess self-understanding of personal mental health problems, self-management, and psychosocial support provided by trained HV used to address them. See the list of the interview questions.

\section{Ethical issues}

This study is a part of the research entitled "Common Health Problem Management of Older Adults in Community". Prior to data collection, an ethical agreement had been granted in 2015 (reference No. 027/2558) from the Committee on Human Rights Related to Research Involving Human Participants, Walailak University, Thailand. Ethical issues during this study process involved the participants' independence, intimacy, and anonymity. The participants were informed of the goal, the study draft, and the voluntary kind of their participation, more importantly reassurance that withdrawal from the study would not affect their healthcare service and their life. The participants gave permission for tape recording of interviews and submitted to the author informed consents to participate in this study. The consent forms and tapes containing data were kept in a safe place and it was destroyed when the study was completed.

\section{Data collection}

To gather in-depth data, first, the researcher carried out an individual weekly interview lasting for 60 to 90 min each time with $\mathrm{V}$ and the trained $\mathrm{HV}$ in a confidential room 4 times, depending on the participants' conditions. The interviews were conducted after $\mathrm{V}$ had been put through the screening and process aimed to manage depressive symptoms and prevent suicide by trained HV for a minimum of 18 months. To probe the participants' responses about preventing depression and suicide challenges, the researcher started the interviews with a common open-ended question and then proceeded with additional questions. The participants got an opportunity, and were encouraged, to talk about events or observations about the study-related issues. The data collection and analysis process were carried out simultaneously for the purpose of developing topics related to the reality of the participants' aspects and experiences of depression and preventing suicide in a rural community. The interviews ended after the themes had been recognized and informational contentment was gained.

\section{Trustworthiness of the study}

Trustworthiness of data and interpretation of this present study was cultivated on the grounds of credibility, transferability, dependability, and conformability. Credibility was established through triangulation consisting of participant checking, peer checking, and a prolonged engagement. Participant checking was done by asking the participants to audit conclusions drawn from the earlier interviews. Peer checking was conducted by 3 authors. A prolonged engagement with the participants within the research field enabled the first author to genuinely earn the participants' trust and a better understanding of the research field. In terms of transferability assurance, the researcher provided a detailed description elucidating life facts of the participants which include their experience, emotion, and context. For attaining dependability and conformability, the researcher team reviewed and approved of the detail of each process as frequently as possible to ultimately attain to the goal of the study.

\section{Data analysis}

Content analysis approach was applied to analyze the data. Data transcriptions were first prepared in Thai. For the participants' responses to each question, the researchers independently analyzed the data by 
http://wjst.wu.ac.th

recognizing and assigning codes to each group. Then, the codes and the latest analysis of the 3 authors were compared. In parts where mutual agreement was not reached, definitions were cleared up and the discussions continued until it was achieved. Each sentence from the participant's observations, interview transcripts, and field notes was analyzed through domain analysis in order to identify emerging themes and categories across interviews from the patient, the trained HV and researcher team. In domain analysis, statements were digested into 3 parts; namely the cover theme or the main concept, the other terms describing the main concept and the relationship between these terms and cover themes.

\section{Results and discussion}

\section{Results}

The psychosocial support of the trained HV reflects a highly dynamic and non-linear process through 4 processes of support as: 1) support and motivation to keep alive, 2) encouragement for a new life, 3) empowerment for life change, and 4) evaluation of the change process as follows.

\section{Supporting and motivating to keep alive}

The trained HV used several methods such as active listening, supporting, and using concern questions on life and death meaning to improve psychological well-being related to emotions, thoughts, perceptions, and behaviors. The most significant factor was based on V's belief. The trained HV gave him a Dharma book featuring a Buddhist monk discussing concepts of death, suffering, loss, disappointment, and the state of uncontrollability that applies to everything on earth. He posited further that "life is not certain, everyone must be faced with suffering, nature's rule is that everything is brought to life, exists, and dies, awareness of this truth helps people deal with life in an understandable, peaceful, and independent way". The Buddhist monk also states that suffering cannot be solved by death and death is sin. Thus, those undergoing suffering should accept it as inevitable and use the wisdom gained to enhance hardiness in coping with daily life. That one should live a life with "goodness", sense of purpose and of self-improvement. V was motivated by this message and consequently made the decision to put the teaching into practice. He was motivated to change his emotions, thoughts, perception, behavior and extended a helping hand to others facing difficulties, especially an older adult group with depression and suicide in the same community, so that his "goodness of giving" may be known and received by him and his family's spirit. A trained HV supported him to change and deal with life in this way.

Another reason behind V's decision to regain his life control is his determination to cease to be his son's burden. He needs a life allowing him to help himself and others with dignity. In addition, he is concerned about the possible side effects of his medication (antidepressants) and his wish to avoid taking this medication for the rest of his life. His final motivation for this change was the promise he gave to his health volunteer to better manage his mental health problems.

\section{Encouraging for a new life}

The trained HV employed active listening, concern questioning for expressing feelings-thoughtsneeds, and encouragement to help $\mathrm{V}$ to make new choices for positive changes based on his belief related to hardiness and growth as goodness. After that, $\mathrm{V}$ made a commitment with the trained $\mathrm{HV}$ to change his life. He read alternative books and consulted with a trained HV and a nurse practitioner to learn more about healthy mental health, positive thinking, creating inner growth, self-talk, increasing self-esteem, regular exercise, stress reduction, relaxation strategies, and raising happiness by giving help to other older adults facing the same suffering in the community. He identifies with the meaning of life and death in a new way. He plans for self-management and the trained HV plans to support his action in order to maintain psychological well-being for managing depression and suicide risk. He then developed an action plan immensely oriented toward his mental health improvement. After that, he reviewed his plan in coordination with his health volunteer, a nurse, and a doctor twice a month. 
http://wjst.wu.ac.th

\section{Empowering for life change}

The phase of coping strategies, V started preparing his own relaxation strategies to control stress, threats as well as coping strategies. He functions on the basis of positive thinking, self-talk, and inner connection to create love, respect, happiness, and freedom by himself and learn to give to others. He eliminated suffering situations from his life. Some healthy patterns of relaxation strategies and stress management emerged such as daily evening walks, listening to and reading Buddhist principles pertaining to thoughts, perceptions, emotions, behaviors, and practice of maintaining positive psychological well-being. Moreover, he also shared his suffering life story to support and help others sufferers in the community. Trained HV paid $\mathrm{V}$ a regular home visit to provide psychosocial support and feedback, monitor and empower $\mathrm{V}$ to manage mental health problems once a week in the first month and twice a month for the next 5 months, followed by monthly monitoring over the course of 18 months. The trained $\mathrm{HV}$ reported results to a nurse practitioner at the community healthcare center. The nurse practitioner further supported the trained HV and $\mathrm{V}$ at home by supporting, empowering, appreciating, and adding in developing the process until they had confidence for changing and collaborating with the psychiatrist at the community hospital.

\section{Evaluating change process}

$\mathrm{V}$ experienced significant improvement in depressive symptoms and suicidal risk in 6-months. His depression medication reduced yet at times he experienced significant happiness. At that time, he was able to discontinue all prescribed medications. $\mathrm{V}$ maintained these outcomes for over 18 months. $\mathrm{V}$ reflected that "This support from the health volunteer brings me back from death to a new life, I would have died if there was no health volunteer at that time" "Staying alive to give to others means a lot to me, it enhances my self-value, hardiness, and goodness". Trained HV encourages him to commit to this positive change and empower $\mathrm{V}$ to share his success story about raising his confidence and self-esteem.

\section{The expressions of trained $\mathrm{HV}$ as a provider}

The trained HV as a provider explained that the positive changes of the patient impacted her internal growth. She experienced self-actualization through the process of psychosocial support with active listening, concern questioning, empowering, and support based on the patient's beliefs connected to hardiness and growth as goodness. As asserted that 'I didn't believe that I could do it, this process inspired me for internal growth" "I realize that having someone who is willing to listen to you and ask you concern questions can make a difference". Furthermore, the trained HV received respect, acceptance, and solid support from the nurse practitioner of the health care center. "Positive development and respect to improve my attitude, confidence, and working skills makes me eager to measure up to standards of care that nurse practitioners have set for me because I know I will never walk alone." These factors assisted the trained HV to help others and assume the role of a health volunteer.

\section{Discussion}

Psychosocial support provided by the trained HV as a dynamic process generated profound impacts on the patient's emotions, thoughts, perceptions, and behaviors. The helping process started with supporting and motivating the patient to keep alive by seeing a new meaning of life and death based on the patient's religious beliefs. Buddhism doctrine reinforced the cognitive process of examining negative thoughts and replacing them with realistic thinking and goals for necessary changes for healthier life [1618]. These strategies are congruent with cognitive therapy focusing on transforming negative thoughts into desired changes and improving mental health $[17,19]$. Buddhists basically believe that suffering is to learn to live a life or as more commonly known in Thailand, "goodness". This religious and cultural belief motivates people to see that suffering is the truth of life, to change thoughts and perceptions, to live a healthy life with no harm, and to help others $[16,18,19]$. The patient of the present study was dramatically motivated by his adoption of this belief playing a significant role in his self-management of his mental health and illness. These results related to another research which revealed that the patient's belief on religious motivates her to change her meaning in her illness experiences and this meaning appears to have supported her efforts to adopt more positive health beliefs and health behaviors [18]. Mental health related religious beliefs are consistent with the common sense model postulating that people have common sense beliefs about their health and illness that guide the way they cope with being healthy and 
http://wjst.wu.ac.th

ill $[17,18,20]$. This process motivates the patient to realize for living in a new way. After that, the trained HV encouraged him to commit and make more choices for a new life. Then, the patient was empowered to take control of his life through a period of home visit by being appreciated and adding in positive developing process until positive changes surfaced. $[10,18]$. In the last process, trained HV evaluated the changing process and encouraged the patient to maintain changes to raise self-esteem, self-connection, happiness, love, freedom, and peace leading to management of depression and suicide [10,20,21]. These 4 processes reflect a highly dynamic and non-linear process of change.

A trained HV's skills in carrying out the process of psychosocial support is crucial for success which encompass active listening, using concern questioning for expressing feelings-through-needs, encouraging patients to make more choices, supporting and empowering [10,22]. These skills develop good relationships, trust, security, confidence serving as curative factors between patient and trained HV that motivate patient to commit to changing $[10,23]$. These skills improve the patient's learning for healthy mental health by means of thinking positively, cultivating inner growth, increasing self-esteem, exercising regularly, reducing stress, employing healthy relaxation strategies, and raising happiness to maintain psychological well-being to manage depression and suicide [10,22,23].

Psychosocial support consisting of the 4 processes and trained HV's skills facilitate the patient to raise hardiness translated into an aspect of personality thereby mitigating the negativity of depression and stress on health [24,25]. Commitment, challenge, and control are 3 components of hardiness [24]. In the present case study, the participant's cognitive processes, emotions, perception, and behavioral strategies are consistent with hardiness [25]. To control his symptoms, V made a commitment to reshaping his thoughts, emotions, perception, and behaviors. This process urged $\mathrm{V}$ to proactively activate self-management by understanding and accepting truths of life, suffering and transforming his perception from hardiness to goodness [18,21,24,25]. He demonstrated abilities to change his self-management by controlling lifestyle, the way of thought and relaxation. Furthermore, $\mathrm{V}$ challenged himself to achieve goals of effective selfmanagement. In this case, facilitating the development of hardiness was useful in helping $\mathrm{V}$ to manage depression, suicide risks and to achieve his desired goal of "goodness".

Psychosocial support for a patient's self-management is also similar to the concept of "selfmanagement support of chronic care model" in that self-management support which refers to the care and encouragement provided to the patient for better understanding of his role in managing his illness, enabling him to make good choices and sustain healthy behaviors requires a collaborative relationship between the patient and health provider [20,21]. This collaborative partnership supports $\mathrm{V}$ in building the skills and confidence he needs to lead an active and fulfilling life [10,20]. In this case study, the ability to engage in and maintain psychosocial support by the trained HV in maintaining V's self-management in caring was affected by his health status (depression and suicide), stress (the suffering from his family' business bankruptcy and poor support), and cultural beliefs. Together, these factors promoted self-management support.

\section{Treatment implications}

This study reflects that mental health professionals can play a critical role and support trained HV in continual early screening and supporting for older adults with mental health and psychiatric problems in rural communities because they can identify and provide psychosocial support to people to manage symptoms of mental distress in older adults with mental illness, cooperate to identify processes that can reduce mental distress and reinforce healthy behaviors. Trained HV are very important in preventing depression and suicide in a rural community despite the limitation of healthcare manpower that is linked with better mental health and health behaviors. Thus, multidisciplinary clinical teams including a trained $\mathrm{HV}$ are important in treating mental illness in rural communities.

\section{Limitations and future research}

As with any case study, the major limitation is the sample size of one, which limits generalization of the findings. Another limitation is that the timeframe may have been too short to evaluate the potential longterm effects of the intervention. However, the data suggest that the psychosocial support process proved beneficial for up to 18 months. In light of these limitations, future studies are needed with a larger sample 
http://wjst.wu.ac.th

size and an extended timeframe to explore this issue more deeply. No study that could be located examined how the type of trained HV's psychosocial support processes may influence each type and level of depression and suicide. Future research should examine how a trained HV's process differs across different levels of depression or suicide, as well as other psychological problems. In addition, research examining the impact of religious and spiritual beliefs on mental health outcomes is also needed.

\section{Conclusions}

The psychosocial support provided by a trained HV proved effective in managing depressive symptoms and suicide attempt. Furthermore, the results tend to be sustained up to 18 months. Therefore, the psychosocial support processes of the trained HV can be suggested in practical guidelines for health personnel in health care centers. While the findings in the present study are promising, more research is needed with a larger sample size over a longer time frame to determine long-term effectiveness of the process and its applicability to other illnesses and population.

\section{Acknowledgements}

This study was supported by the Institute of Research and Development, Walailak University, Thailand (grant WU 58103). The authors are grateful to the participants for their cooperation.

\section{References}

[1] K Bosanquet, N Mitchell, R Gabe, H Lewis, DM Millan, D Ekers, D Bailey and S Gilbody. Diagnostic accuracy of the Whooley depression tool in older adults in UK primary care. $J$. Affect. Disord. 2015; 182, 39-43.

[2] WHO. Depression Fact Sheet. World Health Organization Media Centre, Available at: http://www.who.int/mediacentre/factsheets/fs369/en, accessed July 2016.

[3] M Miravitlles, J Molina, JA Quintano, A Campuzano, J Pérez and C Roncero. Factors associated with depression and severe depression in patients with COPD. Resp. Med. 2014; 108, 1615-25.

[4] World Health Organization. First WHO report on suicide prevention. World Health Organization Media Centre, Available at: http://www.who.int/mediacentre/news/releases/2014/suicideprevention-report/en, accessed May 2017.

[5] N Wongpanarak and S Chaleoykitti. Depression: A significant mental health problem of elderly. $J$. Royal Thai Army Nurs. 2014; 15, 24-31.

[6] Bureau of Mental Health Promotion and Development, Department of Mental Health, Ministry of Public Health. Guidelines for Mental Health Promotion and Prevention in Elderly for Community Hospitals and Sub-district Health Promotion Hospitals. The National Buddhism Office, Bangkok, 2017, p. 1-2.

[7] SS Deshpande, M Gadkari and SS Raje. Screening for depression and its risk factors in geriatric population: A rural community based study. Asian J. Psychiatr. 2011; 4, 284-87.

[8] BA Gaudiano, CA Schofield, C Davis and LS Rifkin. Psychological inflexibility as a mediator of the relationship between depressive symptom severity and public stigma in depression. J. Context. Behav. Sci. 2017; 6, 159-65.

[9] S Shimotsu and N Horikawa. Self-stigma in depressive patients: Association of cognitive schemata, depression, and self-esteem. Asian J. Psychiatr. 2016; 24, 125-9.

[10] S Aekwarangkoon, N Noonil R Petsirasan U Nuampet and C Phonphet. Perception and needs for competency development in depression screening in the elderly community among village health volunteers. Thai J. Nurs. 2017; 66, 36-43.

[11] TG Rhee, BD Capistrant, JC Schommer, RS Hadsall and DL Uden. Effects of depression screening on diagnosing and treating mood disorders among older adults in office-based primary care outpatient settings: An instrumental variable analysis. Prev. Med. 2017; 100, 101-11. 
http://wjst.wu.ac.th

[12] H Oyama and T Sakashita. Long-term effects of a screening intervention for depression on suicide rates among Japanese community-dwelling older adults. Am. J. Geriatr. Psychiatr. 2016; 24, 28796.

[13] Y Ni, JY Tein, M Zhang, Y Yang and G Wu. Changes in depression among older adults in China: A latent transition analysis. J. Affect. Disord. 2017; 209, 3-9.

[14] ES Kim and SH Konrath. Volunteering is prospectively associated with health care use among older adults. Soc. Sci. Med. 2016; 149, 122-9.

[15] M Nicola, L Helen, CG Carolyn, G Linda and B Peter. Effects of befriending on depressive symptoms and distress: systematic review and meta-analysis. Brit. J. Psychiatr. 2010; 196, 96-101.

[16] JM Azorin, A Kaladjian, E Fakra, M Adida, R Belzeaux, E Hantouche and S Lancrenon. Religious involvement in major depression: Protective or risky behavior? The relevance of bipolar spectrum. J. Affect. Disord. 2013; 150, 753-59.

[17] YA Barton, L Miller, P Wickramaratne, M Gameroff and MM Weissman, Religious attendance and social adjustment as protective against depression: A 10-year prospective study. J. Affect. Disord. $2013 ; \mathbf{1 4 6}, 53-7$.

[18] LD Oakley, S Aekwarangkoon and E Ward. Successful holistic management of type 2 diabetes with depression: A very personal story. Holist. Nurs. Pract. 2011; 25, 88-96.

[19] S Grover, S Sarkar, A Bhalla, S Chakrabarti and A Avasthi. Religious coping among self-harm attempters brought to emergency setting in India. Asian J. Psychiatr. 2016; 23, 78-86.

[20] JT Marian, EM Marjolein, M Hilde and L Anneke. Effects of self-management support programmes on activities of daily living of older adults: A systematic review. Int. J. Nurs. Stud. 2016; 61, 23047.

[21] T Zimmermann, E Puschmann, HVD Bussche, B Wiese, A Ernst, S Porzelf, A Daubmann and M Scherer. Collaborative nurse-led self-management support for primary care patients with anxiety, depressive or somatic symptoms: Cluster-randomised controlled trial (findings of the SMADS study). Int. J. Nurs. Stud. 2016; 63, 101-11.

[22] H Chaudhury, M Campo, Y Michael and A Mahmood. Neighbourhood environment and physical activity in older adults. Soc. Sci. Med. 2016; 149, 104-13.

[23] SZ Ivan, A Koyanagi, S Tyrovolas and JM Haro. The association of relationship quality and social networks with depression, anxiety, and suicidal ideation among older married adults: Findings from a cross-sectional analysis of the Irish longitudinal study on aging (TILDA). J. Affect. Disord. 2015; 179, 134-41.

[24] NSK Sadaghiani. The role of hardiness in decreasing the stressors and biological, cognitive and mental reactions. Proc. Soc. Behav. Sci. 2011; 30, 2427-30.

[25] R Delahaij, AWK Gaillard and KV Dam. Hardiness and the response to stressful situations: Investigating mediating processed. Pers. Individ. Dif. 2010; 49, 386-90. 